\title{
Mathematical study of the biodegradation of xenobiotic polymers with experimental data introduced into analysis
}

\author{
M. Watanabe* F. Kawai ${ }^{\dagger}$
}

(Received 27 May 2006; revised 21 April 2007)

\begin{abstract}
A mathematical model for a microbial depolymerization process is analyzed numerically in order to study the biodegradation of a xenobiotic polymer. The weight distribution of a polymer with respect to the molecular weight before and after cultivation of microorganisms are introduced into computational analysis. A time dependent degradation rate is determined by solving an inverse problem, and the transition of the weight distribution is simulated. The result shows that the mathematical model is practically appropriate, and the oxidation is the primary factor in the biodegradation of the polymer. The techniques that can be extended to cover the biodegradation of other polymers are illustrated.
\end{abstract}

${ }^{*}$ Graduate School of Environmental Science, Okayama University, Okayama, JAPAN. mailto: watanabe@ems.okayama-u.ac.jp

${ }^{\dagger}$ Research Institute for Bioresources, Okayama University, Okayama, JAPAN.

See http://anziamj.austms.org.au/V47EMAC2005/Watanabe for this article, (C) Austral. Mathematical Soc. 2007. Published June 6, 2007. ISSN 1446-8735 


\section{Contents}

1 Introduction

C666

2 Incorporation of microbial population growth in exogenous depolymerization model

C669

3 Computational analysis of the inverse problem

C671

4 Simulation of PEG biodegradation

C674

5 Discussion

C679

References

C679

\section{Introduction}

Biodegradation is a practical asset against undesirable accumulation of xenobiotic polymers, and it is an essential factor in environmental protection. Microbial depolymerization processes are generally categorized into either one of two types: exogenous type or endogenous type. In an exogenous depolymerization process, monomer units are split from their terminals stepwise. Examples include the $\beta$-oxidation of polyethylene $(\mathrm{PE})$ and the oxidation of polyethylene glycol (PEG). One finds two primary factors in the microbial depolymerization processes of PE: the gradual weight loss of large molecules due to the $\beta$-oxidation; and the direct consumption or absorption of small molecules by cells. On the other hand, one characteristic of endogenous depolymerization processes is the rapid breakdown of large molecules due to internal cleavages to produce small molecules. Examples include the enzymatic degradation of polyvinyl alcohol (PVA). Mathematical models for those depolymerization processes have been proposed, and studies based on these models conducted. Results of numerical simulations are well in accordance 
with experimental data $[1,2,7,8,9,10,11,13,14,15]$.

Here the analytical and numerical study of exogenous depolymerization processes is extended to cover the biodegradation of PEG. PEG is one of the polyethers whose chemical structures are represented by the expression $\mathrm{HO}(\mathrm{R}-\mathrm{O})_{n} \mathrm{H}$ : for three examples, $\mathrm{PEG}$ is $\mathrm{R}=\mathrm{CH}_{2} \mathrm{CH}_{2}$; polypropylene glycol (PPG) is $\mathrm{R}=\mathrm{CH}_{3} \mathrm{CHCH}_{2}$; and polytetramethylene glycol (PTMG) is $\mathrm{R}=$ $\left(\mathrm{CH}_{2}\right)_{4}$ [3]. Polyethers are utilized as constituents in a number of products including lubricants, antifreeze agents, inks, and cosmetics. Polyethers are either water soluble or oily liquid, and are eventually discharged into the environment through sewage. PEG is produced in the largest quantity among polyethers, and its major part is consumed in the production of nonionic surfactants.

PEG is depolymerized exogenously by liberating $\mathrm{C}_{2}$ compounds, either aerobically or anaerobically $[4,5,12]$. High performance liquid chromatography (HPLC) patterns were analyzed to obtain the initial weight distribution of PEG with respect to the molecular weight, and its weight distribution after the cultivation of a microbial consortium E1. Figure 1 shows the results of the HPLC analysis. The biodegradability of PEG is studied by introducing the weight distribution into the mathematical analysis. We formulate an inverse problem as opposed to an initial value problem to determine the degradation rate for which the solution of the initial value problem satisfies not only the initial weight distribution but also the weight distribution after cultivation. We present a numerical solution of the inverse problem. We also present a result of numerical simulation for the transition of the weight distribution. In previous studies, the degradation rate was assumed to be independent of time $[11,13,14]$. Here a time dependent degradation rate is considered. 


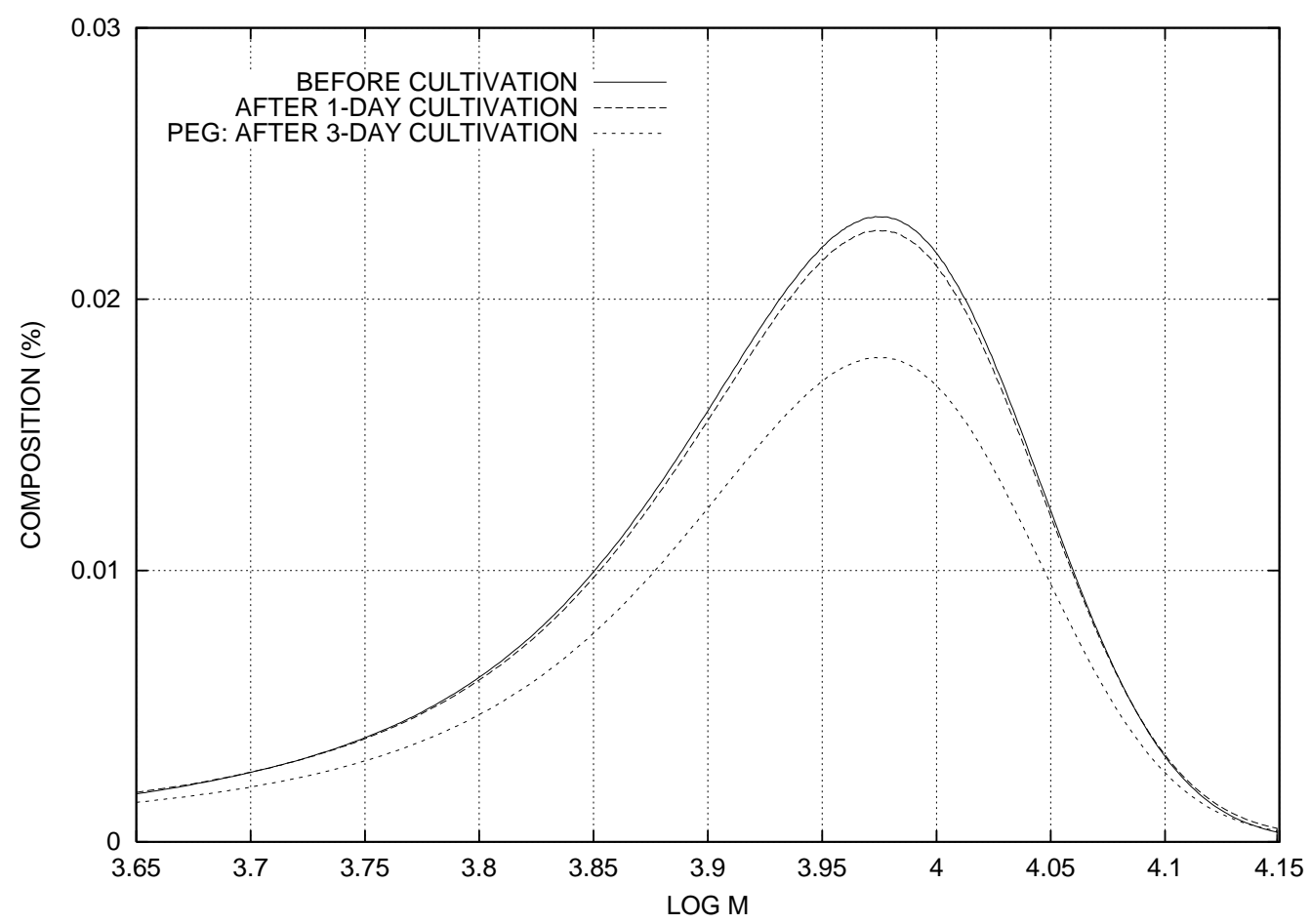

Figure 1: Weight distribution of PEG before and after cultivation of a microbial consortium E1 for one day and three days. 


\section{Incorporation of microbial population growth in exogenous depolymerization model}

The PE biodegradation model is based on two essential factors: the gradual weight loss of large molecules due to the terminal separation ( $\beta$-oxidation); and the direct consumption of small molecules by cells. These assumptions lead to the PE biodegradation model [1, 2, 9]:

$$
\frac{d x}{d t}=-\alpha(M) x+\beta(M+L) \frac{M}{M+L} y,
$$

where $\alpha(M)=\rho(M)+\beta(M)$. Here $t$ and $M$ represent the time and the molecular weight respectively. We call a PE molecule with molecular weight $M$ a $M$-molecule. Then $x=w(t, M)$ represents the total weight of $M$-molecule present at time $t$. The parameter $L$ represents the amount of the weight loss due to the $\beta$-oxidation. The variable $y=w(t, M+L)$ so that $y$ is the total weight of $(M+L)$-molecules present at time $t$. The function $\rho(M)$ represents the direct consumption rate, and the function $\beta(M)$ represents the rate of the weight conversion from the class of $M$-molecules to the class of $(M-L)$-molecules due to the $\beta$-oxidation. The left-hand side of equation (1) represents the rate of change in the total weight of $M$-molecules. The first term on the right-hand side of equation (1) represents the amount lost by the direct consumption and the $\beta$-oxidation in the total weight of $M$ molecules per unit time, and the second term represents the amount gained by the $\beta$-oxidation of $(M+L)$-molecules per unit time.

The weight distribution of $\mathrm{PE}$ with respect to the molecular weight before and after cultivation of fungus Aspergillus sp. AK-3 for three weeks was introduced into analysis to determine the biodegradation rates. Using the degradation rates, the weight distribution after five weeks of cultivation was simulated, and the numerical result seemed acceptable in comparison with 
the experimental result. The model was adequate to PE biodegradation because the degradation rates were allowed to be assumed constant. The reduction rates of total weight over the first three weeks and five weeks were approximately $11.6 \%$ and $18.2 \%$, which shows that the reduction rate was proportional to the cultivation time. For the weight distribution shown in Figure 1, the reduction rates of total weight over the first one day and five days were approximately $1.7 \%$ and $22.2 \%$, which shows that the total weight reduced faster after one day of cultivation. The foregoing discussion suggests that the period for microbial population to reach the steady state was negligible in weeks of cultivation of Aspergillus sp. AK-3 on PE, and that it was allowed to be assumed constant. It also suggests that the microbial population was still in a developing stage in days of cultivation of microbial consortium E1 on PEG.

The mathematical model (1) was originally developed for the PE biodegradation, but it can also be viewed as a general biodegradation model involving exogenous depolymerization processes. In the exogenous depolymerization of PEG, a PEG molecule is first oxidized at its terminal, and then an ether bond is split. It follows that $L=44\left(\mathrm{CH}_{2} \mathrm{CH}_{2} \mathrm{O}\right)$ in the exogenous depolymerization of PEG. It is appropriate for the depolymerization processes over the period after the microbial population reaches the steady state. It has been suggested that the microbial population should be taken into consideration for the period before the microbial population reaches the steady state. The dependence of biodegradability on time was due to the growth of microbial population whose effects on PEG molecules should be uniform regardless of molecular sizes. Then the degradation rate should be proportional to the microbial population, and the model should take the form

$$
\frac{d x}{d t}=-\beta(t, M) x+\beta(t, M+L) \frac{M}{M+L} y,
$$

where

$$
\beta(t, M)=\phi(t) \psi(M) .
$$


The solution $x=w(t, M)$ of (2) is associated with the initial condition

$$
w(0, M)=f(M),
$$

where $f(M)$ is some prescribed function that represents the initial weight distribution. Given the the degradation rate $\beta(t, M)$, the differential equation (2) and the initial condition (3) form an initial value problem to find the unknown function $w(t, M)$. On the other hand, given the initial condition (3) and an additional condition

$$
w(T, M)=g(M),
$$

for some $T>0$, an inverse problem is formulated to determine the degradation rate $\beta(t, M)$ for which the solution $w(t, M)$ of the initial value problem (2) and (3) also satisfies the final condition (4).

\section{Computational analysis of the inverse problem}

The solution $x=w(t, M)$ of the initial value problem (2), (3) is

$$
w(t, M)=e^{-\xi(t) \psi(M)}\left\{f(M)+\frac{M \psi(M+L)}{M+L} \int_{0}^{t} p(M, \psi(M), s) d s\right\},
$$

where

$$
p(M, \eta, s)=e^{\xi(s) \eta} \phi(s) w(s, M+L) .
$$

The condition (4) leads to

$$
g(M)=e^{-\xi(T) \psi(M)}\left\{f(M)+\frac{M \psi(M+L)}{M+L} \int_{0}^{T} p(M, \psi(M), s) d s\right\},
$$

and $\eta=\psi(M)$ is a solution of the equation

$$
\Phi(\eta)=0,
$$


where

$$
\Phi(\eta)=e^{-\xi(T) \eta}\left\{f(M)+\frac{M \psi(M+L)}{M+L} \int_{0}^{T} p(M, \eta, s) d s\right\}-g(M) .
$$

It follows that

$$
\Phi^{\prime}(\eta)=-e^{-\xi(T) \eta}\left\{\xi(T) f(M)+\frac{M \psi(M+L)}{M+L} \int_{0}^{T} q(M, \eta, s) d s\right\}
$$

where

$$
q(M, \eta, s)=[\xi(T)-\xi(s)] p(M, \eta, s) .
$$

In order to solve the inverse problem numerically, the interval $[a, b]$ is covered with the intervals of length $L:[a+i L, a+(i+1) L], i=0, \ldots, l-$ 1 , extending the definition of $f(M)$ and $g(M)$ if necessary. The positive integer $l$ satisfies the condition

$$
\frac{b-a}{L} \leq l<\frac{b-a}{L}+1
$$

Set

$$
M_{i, j}=a+i L+j \delta M, \quad i=0,1, \ldots, l-1, \quad j=0,1, \ldots, m \quad\left(\delta M=\frac{L}{m}\right),
$$

where $m$ is a positive integer. Denote by $\psi_{i, j}$ approximate values of $\psi\left(M_{i, j}\right)$. Set

$$
t_{k}=k \delta t, \quad k=0,1,2, \ldots, n \quad\left(\delta t=\frac{T}{n}\right),
$$

where $n$ is a positive integer. Denote by $w_{i, j, k}$ approximate values of $w\left(t_{k}, M_{i, j}\right)$. Given $w_{i+1, j, k}, j=m-1, m-2, \ldots, 0, k=0,1, \ldots, n$, and $\psi_{i+1, j}, j=$ $m-1, m-2, \ldots, 0, \eta=\psi_{i, j}$ is a solution of the equation

$$
\Phi_{i, j}(\eta)=0,
$$


where

$$
\Phi_{i, j}(\eta)=e^{-\xi(T) \eta}\left(f_{i, j}+\frac{\delta t \psi_{i+1, j} M_{i, j}}{2 M_{i+1, j}} \sum_{k=1}^{n} r_{i, j, k}(\eta)\right)-g_{i, j}
$$

where

$$
r_{i, j, k}(\eta)=e^{\xi\left(t_{k-1}\right) \eta} \phi\left(t_{k-1}\right) w_{i+1, j, k-1}+e^{\xi\left(t_{k}\right) \eta} \phi\left(t_{k}\right) w_{i+1, j, k}
$$

and $f_{i, j}=f\left(M_{i, j}\right), g_{i, j}=g\left(M_{i, j}\right)$.

The numerical process is started at $i=l-1$ setting appropriate values of $w_{l, j, k}, j=0,1, \ldots, m-1, k=0,1, \ldots, n$, and marched backward: $i=$ $l-1, l-2, \ldots, 0$. At each step, a numerical solution $\eta=\alpha_{i, j}$ of $(8)$ is obtained by Newton's method for $j=m-1, m-2, \ldots, 0$. Once an approximate solution $\eta=\alpha_{i, j}$ of the equation (8) is found, approximate values $w_{i, j, k}$ of $w\left(t_{k}, M_{i, j}\right)$ are

$$
w_{i, j, k}=e^{-\xi\left(t_{k}\right) \eta}\left(f_{i, j}+\frac{\delta t \beta_{i+1, j} M_{i, j}}{2 M_{i+1, j}} \sum_{l=1}^{k} r_{i, j, l}(\eta)\right),
$$

with $\eta=\alpha_{i, j}$ for $k=0,1, \ldots, n$.

Suppose that $N(t)$ is the population of a species at time $t$ and assume that $N$ is a solution of

$$
\frac{d N}{d t}=r N\left(1-\frac{N}{K}\right)
$$

where $r$ and $N$ are positive constants, which models a self-limiting process called logistic growth in a population [16]. The solution of the equation, which satisfies the initial condition $N(0)=N_{0}$, is

$$
N(t)=\frac{N_{0} K e^{r t}}{K+N_{0}\left(e^{r t}-1\right)}
$$


The time factor $\phi(t)$ of the degradation rate is assumed to be proportional to the microbial population. Letting $c=K-1$ and assuming that $\phi(0)=1$, the following expression for $\phi(t)$ is obtained from the solution of the equation:

$$
\phi(t)=\frac{1+c}{1+c e^{-r t}},
$$

where $c$ and $r$ are positive constants. It follows that

$$
\xi(t)=\int_{0}^{t} \phi(s) d s=\frac{1+c}{r} \log \left(\frac{1+c e^{-r t}}{(1+c) e^{-r t}}\right) .
$$

The inverse problem was solved numerically using this function $\phi(t)$ for $r=$ 0.001 and $c=1000$ setting $a=10^{3.63} \approx 4266, b=10^{4.15} \approx 14125, L=44$, $m=10, l=225$, and $n=1200$. Approximate values of $\psi(M)$ were obtained numerically based on the data shown in Figure 1 using the HPLC data before and after cultivation of a microbial consortium E1 for three days. Figure 2 shows the numerical result.

\section{Simulation of PEG biodegradation}

In the process of evaluating the exogenous degradation rate, the approximate values $w_{i, j, k}$ of $w(t, M)$ at $t=t_{k}$ and $M=M_{i, j}$ are also evaluated. On the other hand, once the approximate values of the total consumption rates and the $\beta$ oxidation rates are given, the initial value problem (1) and (3) can be solved directly to see how well the numerical results and the experimental results agree. Here the initial value problem was solved numerically with techniques developed previously $[2,9,10]$.

Choose a positive integer $N$ and set

$$
M_{i}=a+i \Delta M, \quad i=0,1,2, \ldots, N \quad\left(\Delta M=\frac{b-a}{N}\right) .
$$




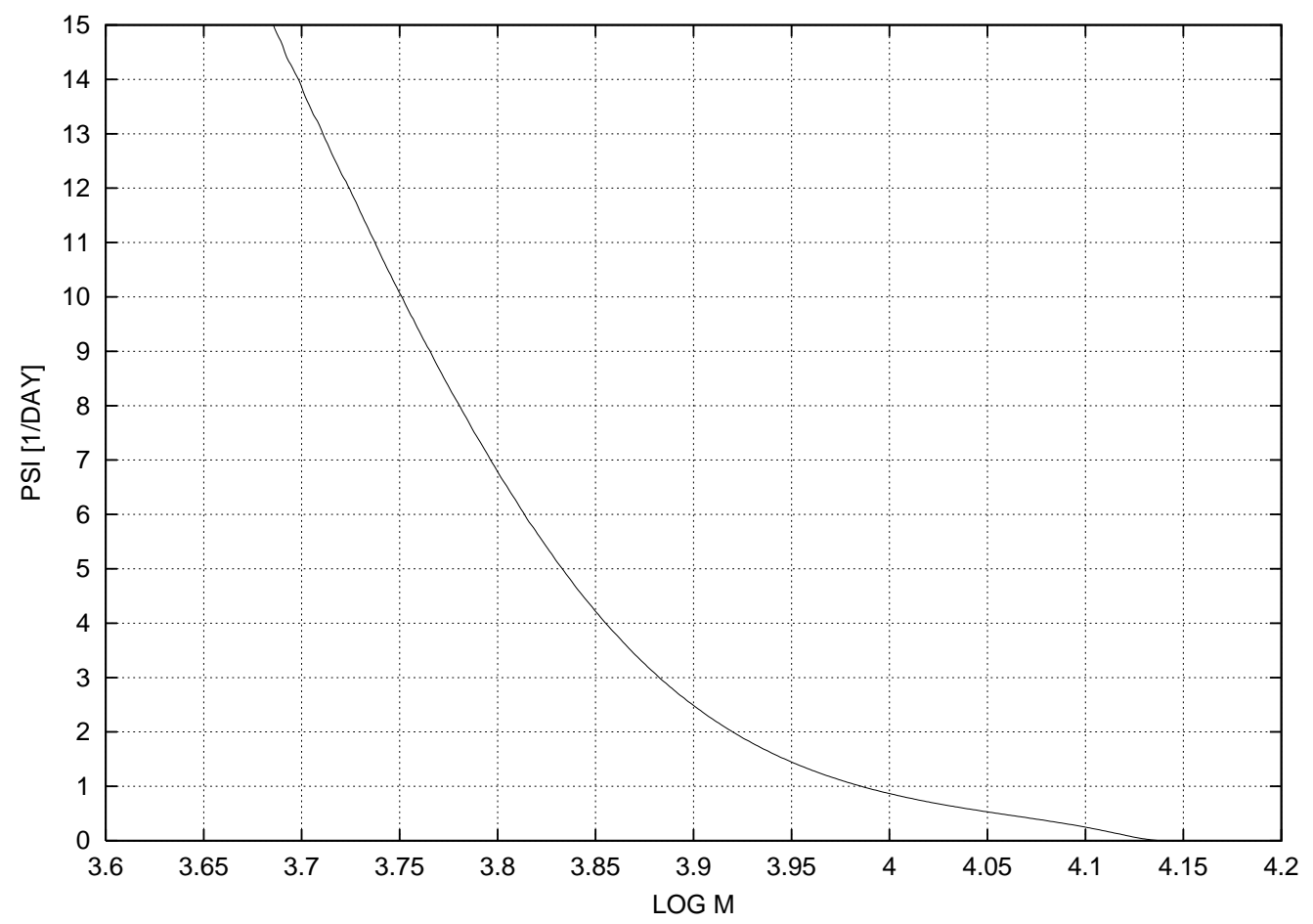

Figure 2: Approximate values of $\psi(M)$ based on the HPLC data before and after cultivation of a microbial consortium E1 for three days. 
An approximate solution of the differential equation (1) at $M=M_{i}$ is denoted by $w_{i}=w_{i}(t) i=0,1,2, \ldots, N$. There is a non-negative integer $K$ and a constant $R$ such that $L=K \Delta M+R, 0 \leq R<\Delta M$, and such that the inequalities

$$
M_{i+K} \leq M_{i}+L<M_{i+K+1}
$$

hold. Then approximate values of $w\left(t, M_{i}+L\right)$ and $\beta\left(M_{i}+L\right)$ are obtained using

$$
\begin{aligned}
w\left(t, M_{i}+L\right) & \approx\left(1-\frac{R}{\Delta M}\right) w\left(t, M_{i+K}\right)+\frac{R}{\Delta M} w\left(t, M_{i+K+1}\right), \\
\beta\left(t, M_{i}+L\right) & =\phi(t) \psi\left(M_{i}+L\right) \\
& \approx \phi(t)\left\{\left(1-\frac{R}{\Delta M}\right) \psi\left(M_{i+K}\right)+\frac{R}{\Delta M} \psi\left(M_{i+K+1}\right)\right\} .
\end{aligned}
$$

Substituting these expressions in the differential equation (2) and setting $M=M_{i}$, we obtain the linear system

$$
\frac{d w_{i}}{d t}=\phi(t)\left(-\alpha_{i} w_{i}+\beta_{i} w_{i+K}+\gamma_{i} w_{i+K+1}\right), \quad i=0,1,2, \ldots, N .
$$

Here the coefficients

$$
\begin{aligned}
\alpha_{i} & =\psi\left(M_{i}\right), \quad \beta_{i}=\sigma_{i} \frac{M_{i}}{M_{i}+L}\left(1-\frac{R}{\Delta M}\right), \quad \gamma_{i}=\sigma_{i} \frac{M_{i}}{M_{i}+L} \cdot \frac{R}{\Delta M}, \\
\sigma_{i} & =\left(1-\frac{R}{\Delta M}\right) \psi\left(M_{i+K}\right)+\frac{R}{\Delta M} \psi\left(M_{i+K+1}\right) .
\end{aligned}
$$

Approximate values of the degradation rates $\psi\left(M_{i}\right)$ are obtained from the numerical solution of the inverse problem by linear approximation.

For all sufficiently large $M$, the oxidation rate becomes zero. In particular, we assume that the last two terms on the right-hand side of the equation (9) are absent when $i+K$ exceeds $N$, so that the system (9) becomes a closed system to be solved for unknown functions $w_{i}=w_{i}(t), i=0,1,2, \ldots, N$. In view of the condition (3), these functions are subject to the initial condition

$$
w_{i}(0)=f_{i}=f\left(M_{i}\right) .
$$




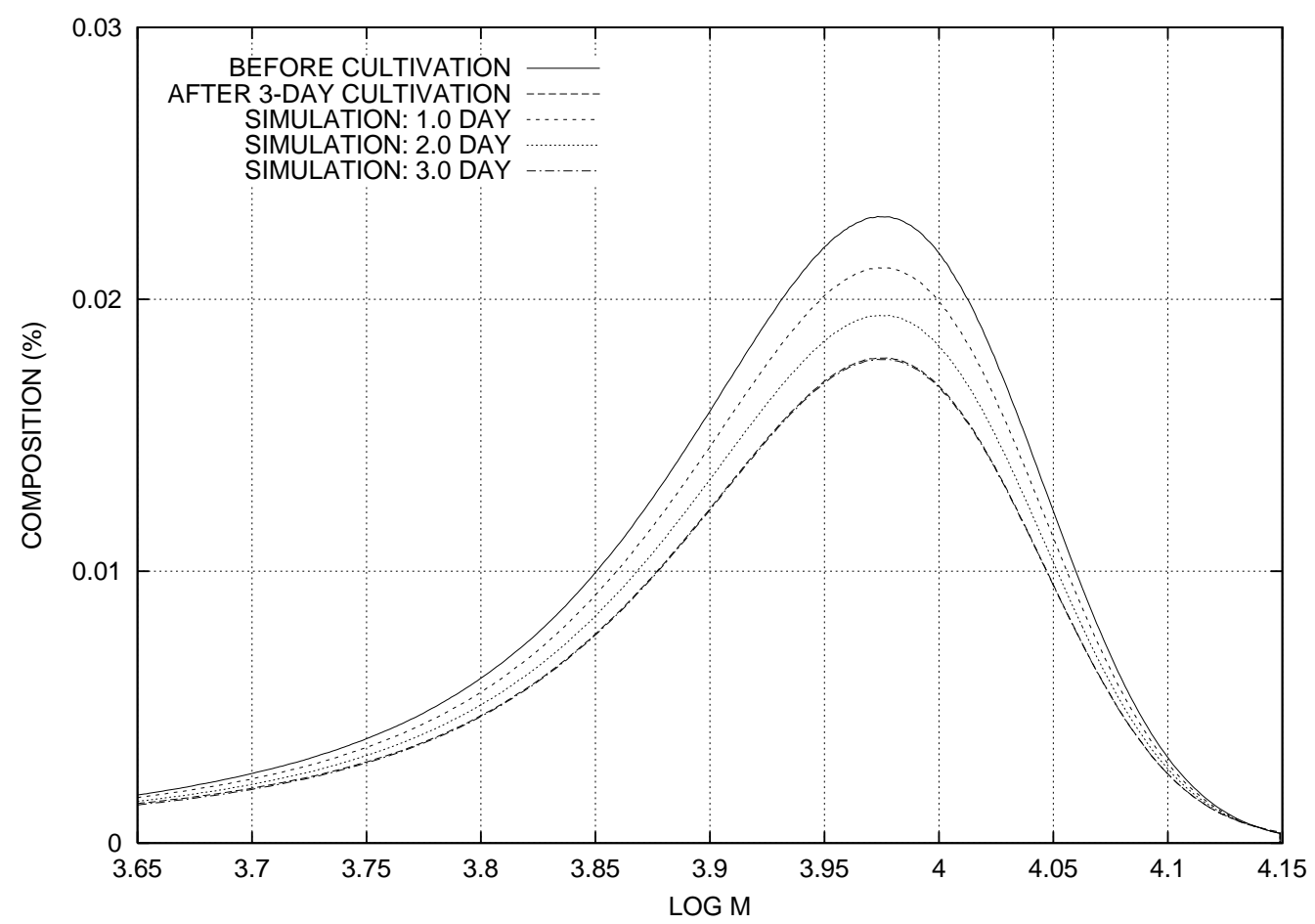

FiguRE 3: Transition of the Weight distribution of PEG during three day cultivation of the microbial consortium E1.

Given the initial weight distribution shown in Figure 1 and the corresponding degradation rate shown in Figure 2, the initial value problem (9) and (10) was solved numerically implementing the fourth order Adams-BashforthMoulton predictor-corrector in PECE mode in conjunction with the RungeKutta method to generate approximate solutions in the first three steps [6] by using $N=10000$, and a time interval $\Delta t=0.0025$. Figure 3 shows the numerical result. It shows the transition of the weight distribution during cultivation of the microbial consortium E1 for three days. Figure 4 shows the numerical result and the experimental results for the weight distribution after one day cultivation of the microbial consortium E1. 


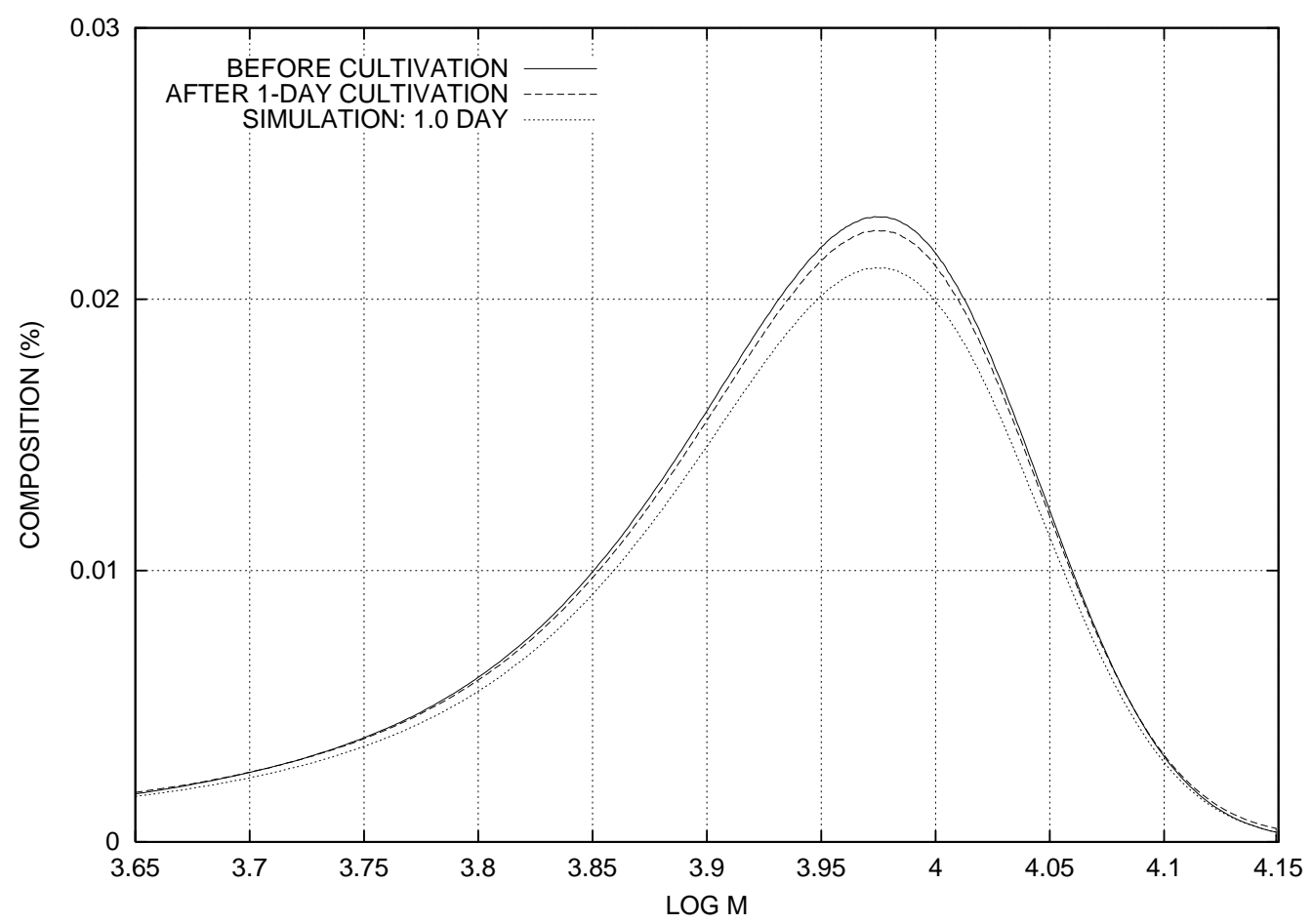

FiguRE 4: Transition of the Weight distribution of PEG during one day cultivation of the microbial consortium E1. 


\section{Discussion}

The time dependent degradation rate has been considered in the mathematical modeling of the biodegradation of exogenous type. It is possible to solve the inverse problem numerically to obtain the time dependent degradation rate introducing the experimental data into the analysis. The transition of the weight distribution can be simulated with the time dependent degradation rate.

Acknowledgements: The authors thank Ms Y. Shimizu (Research Institute for Bioresources, Okayama University) for her technical support. This work was supported in part by a JSPS (MEXT) Grant-in-Aid for Scientific Research (C) (16540106).

\section{References}

[1] Fusako Kawai, Masaji Watanabe, Masaru Shibata, Shigeo Yokoyama, Yasuhiro Sudate, Experimental analysis and numerical simulation for biodegradability of polyethylene, Polymer Degradation and Stability 76 (2002) 129-135. doi:10.1016/S0141-3910(02)00006-X C667, C669

[2] Masaji Watanabe, Fusako Kawai, Masaru Shibata, Shigeo Yokoyama, Yasuhiro Sudate, Computational method for analysis of polyethylene biodegradation, Journal of Computational and Applied Mathematics, Volume 161, Issue 1, 1 December 2003, 133-144. doi:10.1016/S0377-0427(03)00551-X C667, C669, C674

[3] Fusako Kawai, Biodegradability and chemical structure of polyethers, Kobunshi Ronbunshu, 50(10), 775-780 (1993) (in Japanese). C667 
[4] Fusako Kawai, Breakdown of plastics and polymers by microorganisms, Advances in Biochemical Engineering/Biotechnology, Vol. 52, 151-194 (1995). C667

[5] Fusako Kawai, Microbial degradation of polyethers, Applied Microbiology and Biotechnology (2002) 58:30-38. doi:10.1007/s00253-001-0850-2 C667

[6] J. D. Lambert, Computational Methods in Ordinary Differential Equations, John Wiley and Sons, Chichester, 1973. C677

[7] Masaji Watanabe, Fusako Kawai, Numerical Simulation for Enzymatic Degradation of Poly (vinyl Alcohol), Polymer Degradation and Stability, Volume 81, Issue 3, 2003, 393-399. doi:10.1016/S0141-3910(03)00122-8 C667

[8] Masaji Watanabe and Fusako Kawai, Analysis of polymeric biodegradability based on experimental results and numerical simulation, Environmental Research and Control (2003), 25, 25-32 (In Japanese). C667

[9] Fusako Kawai, Masaji Watanabe, Masaru Shibata, Shigeo Yokoyama, Yasuhiro Sudate, Shizue Hayashi, Comparative study on biodegradability of polyethylene wax by bacteria and fungi, Polymer Degradation and Stability 86 (2004), 105-114. doi:10.1016/j.polymdegradstab.2004.03.015 C667, C669, C674

[10] Masaji Watanabe, Fusako Kawai, Masaru Shibata, Shigeo Yokoyama, Yasuhiro Sudate, Shizue Hayashi, Analytical and computational techniques for exogenous depolymerization of xenobiotic polymers, Mathematical Biosciences 192 (2004) 19-37. doi:10.1016/j.mbs.2004.06.006 C667, C674

[11] Masaji Watanabe, Fusako Kawai, Analysis of biodegradability for polyethylene glycol via numerical simulation, Environmental Research and Control 26 (2004), 17-22 (in Japanese). C667 
[12] Fusako Kawai, Xenobiotic polymers, in: T. Imanaka, ed., Great Development of Microorganisms, (NTS. Inc., Tokyo, 2002) 865-870 (in Japanese). C667

[13] Masaji Watanabe, Fusako Kawai, Numerical and experimental Study on Mechanism of Microbial Depolymerization, (International Symposium on Numerical Simulation of Environmental Problems), (November 22-23, 2004 at Faculty of Environmental Science and Technology, Okayama University), Journal of Environmental Science and Technology (Special Edition), 17-29. C667

[14] Masaji Watanabe, Fusako Kawai, Numerical simulation of microbial depolymerization process of exogenous type, Proceedings of the 2004 International Conference on Computational Techniques and Applications, Melbourne, Australia in September 2004, Editors Rob May and A. J. Roberts, ANZIAM J. 46(E) pp.C1188-C1204, 2005. http://anziamj . austms .org.au/V46/CTAC2004/Wata [November 8, 2005] C667

[15] Masaji Watanabe, Fusako Kawai, Mathematical modelling and computational analysis for enzymatic degradation of xenobiotic polymers, Applied Mathematical Modelling 30 (2006) 1497-1514. doi:10.1016/j.apm.2005.12.011 C667

[16] James D. Murray, Mathematical Biology, Second, Corrected Edition, Springer-Verlag, Berlin, 1989. C673 